\title{
Pirólisis de purines de cerdo utilizando TG-DSC
}

\author{
Violeta Quispe, Javier Ábrego, María Benita Murillo, Gloria Gea, María Atienza \\ Grupo de Procesos Termoquímicos (GPT) \\ Instituto de Investigación en Ingeniería de Aragón (I3A). \\ Universidad de Zaragoza, Mariano Esquillor s/n, 50018, Zaragoza, Spain. \\ Tel. +34-976761880, Fax +34-976761880, e-mail: vquispe@unizar.es
}

\begin{abstract}
El uso de técnicas instrumentales como la termogravimetria (TG) y la calorimetría (DSC) ha sido propuesto en el proceso de pirólisis de purines de cerdo bajo diferentes condiciones experimentales permitiendo obtener información fundamental para su aplicación en la adecuada valorización energética y posible nueva via de tratamiento sostenible.
\end{abstract}

\section{Introducción}

España es el segundo productor de porcino de la Unión Europea y su producción anual de purines oscila entre los 35 y 50 millones de toneladas. La mayor parte se aplica al suelo pero dada la elevada producción de los mismos y la contaminación que causa al suelo y en el agua, es preciso buscar otras formas de tratamiento. Entre ellas aparece la pirólisis como una posible opción de valorización energética dado el poder calorífico de este residuo.

La viabilidad técnica y económica depende fundamentalmente del balance energético del proceso (energía consumida y energía almacenada de los productos obtenidos) y generalmente de las posibilidades de aprovechamiento energético y material de los productos. Esto a su vez se viene influenciado por las condiciones de operación [1].

La Termogravimetría (TG) y la calorimetría (DSC) son técnicas instrumentales que suministran información significativa sobre el comportamiento pirolítico de la biomasa, asi como la cinética de descomposición, su mecanismo de reacción e identificar procesos exotérmicos o endotérmicos [2].

El análisis termogravimétrico (TG) proporciona información con respecto a la curva de pérdida de peso en función de la temperatura y tiempo. Mientras que la calorimetría calcula la energía absorbida o liberada por una muestra, a partir del flujo calorífico diferencial (DSC). Con la combinación de ambos métodos, se podría proporcionar información valiosa para poder empezar a plantear estudios a mayor escala del proceso aplicado a este material [3].
Aun así existe escasa información a nivel nacional e internacional, sobre el procesado termoquímico del purín por lo cual este grupo de investigación se centra en la investigación sobre este campo.

En este estudio se presentan los resultados de experimentos realizados en una Termobalanza empleando purines de cerdo que provienen digeridos anaeróbicamente $\mathrm{y}$ en base seca. Los resultados obtenidos fueron evaluados $\mathrm{y}$ comparados con otro residuo que se estudia en el mismo grupo de investigación desde hace más tiempo.

\section{Metodo Experimental}

Se utilizó una termobalanza de horno tipo Std SiC S y crisol de Platino-Rodio. Antes de introducir las muestras, se molieron en un mortero de ágata. En la Tabla 1 se muestran las características y análisis inmediato del purín.

Tabla 1. Características del purín

\begin{tabular}{|l|c|c|c|c|}
\hline \multicolumn{1}{|c|}{ Análisis inmediatos de ADPC } & Valores \\
\hline \multicolumn{2}{|l|}{ Poder calorífico Superior (MJ/Kg) } & \multicolumn{2}{|c|}{12,4} \\
\hline Humedad (\% en masa) & \multicolumn{2}{|c|}{5,0} \\
\hline Cenizas (\% en masa) & \multicolumn{2}{|c|}{29,4} \\
\hline Volátiles (\% en masa) & \multicolumn{2}{|c|}{54,7} \\
\hline $\begin{array}{l}\text { Composición } \\
\text { elemental \% en masa }\end{array}$ & 6,1 & 26,3 & 4,6 & 5,8 \\
\hline
\end{tabular}

Tabla 2. Condiciones experimentales de operación

\begin{tabular}{|c|c|c|c|}
\hline Experimento & $\begin{array}{c}\text { Masa } \\
\text { de } \\
\text { purín } \\
(\mathbf{m g})\end{array}$ & $\begin{array}{c}\text { Tamañ } \\
\text { o de } \\
\text { Partícu } \\
\text { la }(\boldsymbol{\mu m})\end{array}$ & $\begin{array}{c}\text { Velocidad } \\
\mathbf{d e} \\
\text { calentamie } \\
\text { nto } \\
{ }^{\circ} \mathbf{C} / \mathbf{m i n}\end{array}$ \\
\hline 1 & 20 & $50-100$ & 5 \\
\hline 2 & 20 & $\leq 50$ & 10 \\
\hline 3 & 20 & $50-100$ & 10 \\
\hline 4 & 31 & $50-100$ & 10 \\
\hline
\end{tabular}


Los experimentos dinámicos se llevaron a cabo en condiciones de atmósfera inerte, con un intervalo de temperatura entre $25^{\circ} \mathrm{C}$ hasta $800^{\circ} \mathrm{C}$ y flujo de gas de $70 \mathrm{ml}(\mathrm{STP}) / \mathrm{min}$. En la tabla 2 se muestra los experimentos realizados a las diferentes condiciones de operación.

\section{Resultados}

En la Figura a) se muestra la variación de la pérdida de peso del material frente a la temperatura para los dos residuos analizados, purines y lodos de depuradora. La perdida de peso total obtenida hasta $800{ }^{\circ} \mathrm{C}$ es un $60 \%$ y $55 \%$ en masa, respectivamente. Estas diferencias podrían ser debido en parte a un menor contenido de cenizas y un mayor contenido en volátiles de los purines.

Las máximas pérdidas de peso se producen a temperaturas inferiores en el purín que en el lodo, en el purín se produce aproximadamente entre 200 y $300^{\circ} \mathrm{C}$ y en el lodo entre $220-380{ }^{\circ} \mathrm{C}$. Solo en el caso del purín hay una perdida de peso a temperaturas altas (en torno a $700^{\circ} \mathrm{C}$ ) que podría ser debido en parte a la descomposicióntérmica de algún compuestoinorgánico.

En la Figura b) se presentan la curva de DSC y DTG del Experimento $n^{\circ} 3$ del purín. Se observa que, los picos de perdida de peso coinciden con los picos de cambio de energía, mostrando que los procesos de descomposición que ocurren durante la pirólisis de purines son mayoritariamente endotérmicos.

Para llevar a cabo la pirólisis de purines hasta 550 ${ }^{\circ} \mathrm{C}$ la curva de DSC nos indica que se requiere un aporte energético entorno a $0.8 \mathrm{MJ} / \mathrm{kg}$.

La Figura c) representa las curvas TG y DTG obtenidas para los purines bajo las diferentes condiciones de operación analizadas (Tabla 2). Se observa mayores velocidades de descomposición para la mayor velocidad de calentamiento analizada. Sin embargo, apenas se aprecian diferencias entre los experimentos realizados con distinta cantidad de masa lo que indica que $20 \mathrm{mg}$ es una cantidad adecuada para reducir problemas difusionales.

\section{Conclusiones}

Los resultados nos indican que, mediante la pirólisis, se puede lograr reducir estos residuos hasta un 60 y un 55\% en masa, respectivamente. Aunque los lodos y los purines son materiales que podríamos considerar similares, ya que ambos proceden de un proceso de digestión anaerobia de la materia orgánica de un residuo con elevado contenido en agua (superior al $95 \%$ en masa), se puede observar que su comportamiento en la termogravimetría es distinto, especialmente a altas temperaturas, lo que nos indica que probablemente su composición inorgánica sea diferente.

El proceso de pirólisis de los purines hasta una temperatura de $550^{\circ} \mathrm{C}$ es un proceso endotérmico con necesidades energéticas entorno a $0.8 \mathrm{MJ} / \mathrm{kg}$.

\section{Agradecimientos}

Los autores agradecen al Proyecto JIUZ-2013-TEC01 financiado por la Universidad de Zaragoza. Violeta Quispe agradece al grupo de investigación del GPT y al Banco Santander por el financiamiento predoctoral otorgado.

\section{Referencias}

[1]. FANG, H., WEIMING, Y. and XUEYUAN, B. Investigation on caloric requirement of biomass pyrolysis using TG-DSC analyzer. Energy Conversion and Management. 2006, 47(15), 24612469.

[2]. SHADANGI, K. P. and MOHANTY, K. Kinetic study and thermal analysis of the pyrolysis of nonedible oilseed powders by thermogravimetric and differential scanning calorimetric analysis. Renewable Energy. 2014, 63, 337-344.

[3]. CONESA J.A., URUENA, A. and DIEZ, D. Corn stover thermal decomposition in pyrolytic andoxidant atmosphere. Journal of Analytical and Applied Pyrolysis. 2014, 106, 132-137. 


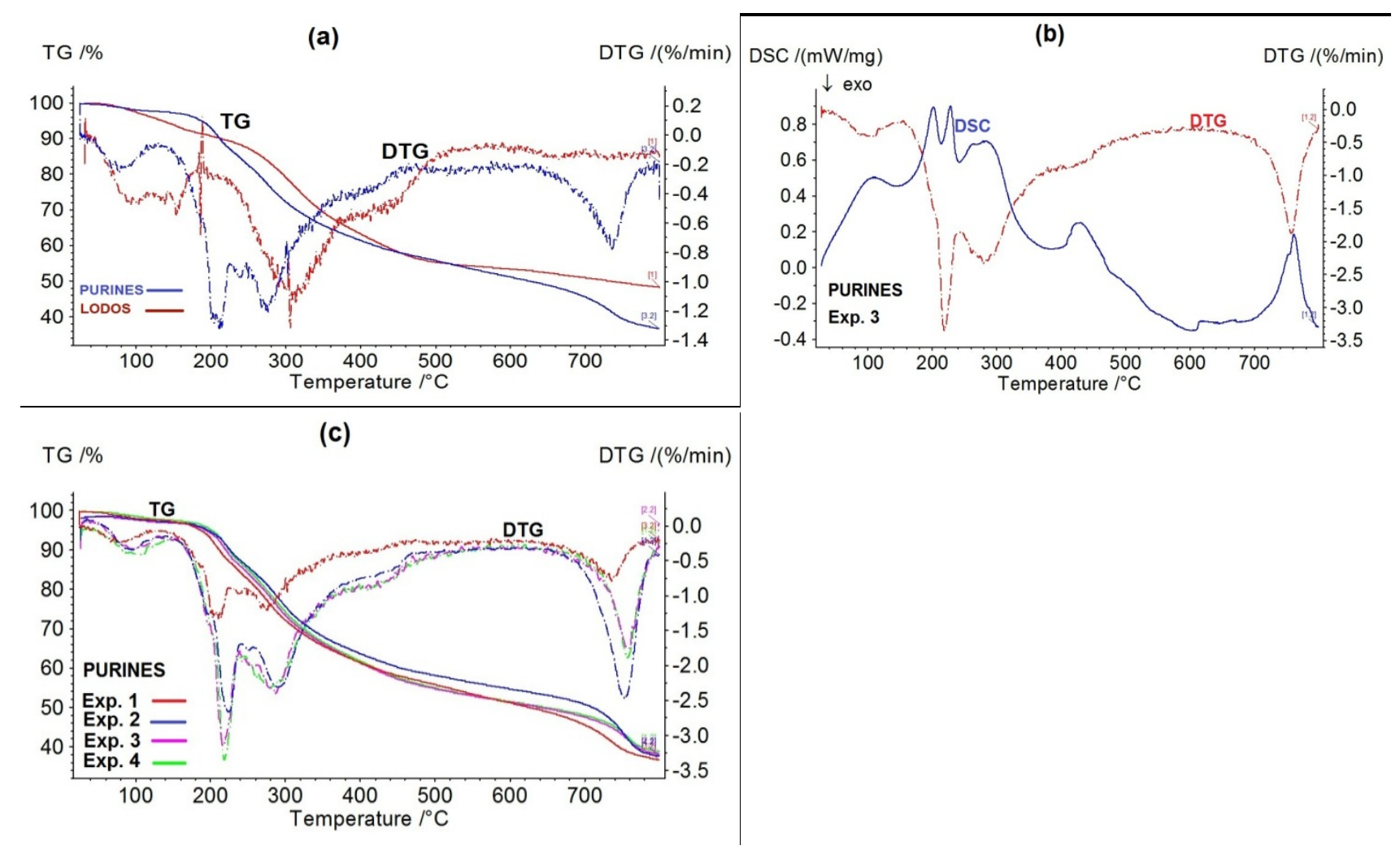

Figura 1: a) Comparación TG y DTG entre resultados de purín y lodo; b) Relación entre DSC y DTG del experimento 3 de los purines y c) Resultados de TG y DTG de los purines a diferentes condiciones. 